von Karman Institute for Fluid Dynamics

Lecture Series 1997-02

\title{
COMPUTATIONAL FLUID DYNAMICS
}

March $3-7,1997$

3D MULTIGRID ON PARTIALLY ORDERED SETS OF GRIDS

P.W. Hemker. B. Koren; J. Noordmans

CWI, The Netherlands 



\title{
3D Multigrid on Partially Ordered Sets of Grids
}

\author{
P.W. Hemker, B. Koren and J. Noordmans \\ CWI, P.O. Box 94079, 1090 GB Amsterdam, The Netherlands
}

\begin{abstract}
In this paper we discuss different possibilities of using partially ordered sets of grids in multigrid algorithms. Because, for a classical sequence of regular grids the number of degrees of freedom grows much faster with the refinement level for $3 \mathrm{D}$ than for $2 \mathrm{D}$, it is more difficult to find sufficiently effective relaxation procedures. Therefore, we study the possibility of using different families of (regular rectangular) grids.

Semi-coarsening is one technique in which a partially ordered set of grids is used. In this case still a unique fine-grid discrete problem is solved. On the other hand, sparse grid techniques are more efficient if we compare the accuracy obtained with the number of degrees of freedom used. However, in the latter case it is not always feasible to identify an appropriate discrete equation that should be solved. The different approaches are compared.

The relation between the different approaches is described by looking at hierarchical bases and by considering full approximation (FAS). We show that in some cases the 3D situation is essentially more difficult than the 2D case. We also describe different multigrid strategies. Numerical results are given for a transonic: Euler-flow over the ONERA M6-wing.
\end{abstract}

Note: In essence, this paper will be published in the Procedings of the Fifth European Multigrid Conference, Birkhäuser, Basel.

\section{Introduction}

Classical multigrid algorithms are based on a sequence of grids, and a sequence of finite dimensional function spaces is associated with it. There is a natural ordering. On the finest grid a discretisation is given and on the coarser grids less accurate discretisations of the same problem accelerate the solution process for the finest problem. This approach is used in two as well as in three dimensions $(d=2,3)$. For illustrative purposes it can also be used in one dimension $(d=1)$.

In the most common approach the secuence of approximating function spaces is nested. Often the coarsest grid consists of a small number of coarse rectangular blocks. A next finer level is obtained by dividing each block in equal parts in each of the coordinate directions, so that for all blocks $2^{d}$ new blocks are created. For higher dimensional problems, the disadvantage is clear: for each next finer level the number of blocks multiplies by $2^{d}$. This implies that only a small portion of the possible error modes on a grid can be represented on the coarser grids. 
The result is that in 3D problems the classical MG method may appear less effectire than in 2 dimensions. Particular problems arise for strongly anisotropic and convection dominated problems, where the so-called "wash board frequencies" (frequencies that are rapidly varying in one and slowly in another direction) may be difficult to control.

The classical cure against these difficulties is the choice of powerful relaxation algorithms, e.g., line or plane relaxations. However, not only the more complex implomentation of these smoothers, but also the large number of possible line and plane combinations makes it a hard job to develop methods that are robust and efficient for general equations.

Another disadvantage of dividing a cube into 8 smaller cubes are the very large systems of linear equations that are found already for a relatively small number of levels. It is the "curse of 3D" that $n^{3}$ is already large -for computer resources- when $n$ is still modest.

Another approach that can be used is semi-coarsening. Here, grids are refined hy halving (or doubling) the mesh size in one direction only $[13,14,16]$. Now the grids do not make an ordered sequence. The family of grids is only partially ordered. In a semi-coarsening algorithm there is a finest grid for which the solution is eventually found. Again, discrete problems on the family of coarser grids are solved to accelerate the solution process. In this way the coarse-grid-correction in the MG algorithm becomes morc complex, but simpler relaxation procedures can be used.

Another approach that makes use of a similar family of partially ordered grids is Zenger's combination technique [3]. Here, essentially a number of independent discreto problems is solved on a subset chosen from the partially ordered set of grids. By extrayolation these discrete solutions are combined to a discrete approximation of the solution on the finest grid (for which no discrete system has to be solved).

Zenger and his co-workers showed that under conditions such an approximation may result in an approximation error which is $\mathcal{O}\left(2^{-d n} n^{d-1}\right)$, whereas the solution is reprosented by $\mathcal{O}\left(n^{d-1} 2^{n}\right)$ degrees of freedom only. This result makes this sparse grid ronresentation of the approximate solution most efficient.

In the present paper we show that in some respects the sparse grid representation in more cumbersome and may give rise to particular difficulties in the $3 \mathrm{D}$ case. We show how some of these can be relieved by taking a representation with $\mathcal{O}\left(n^{2} 4^{n}\right)$ degress of freedom. For a larger number of levels this representation is still much more efficient than the usual $\mathcal{O}\left(8^{n}\right)$ methods.

In Section 2 we show an essential difference betwen the 2D and the 3D case for the linear elliptic second order equation, and in Section 3, as an example of a system of uon-linear equations, we show some results for multiple semi-coarsened and sparse-grid multigrid algorithms for the 3D Euler equations.

\section{Linear elliptic problems}

Let $\Omega=(0,1)^{d} \subset \mathbb{R}^{d}$ be the $d$-dimensional unit cube with boundary $\Gamma$. To identify a grid on $\Omega$ we use a multi-integer notation, $\mathbf{k}=\left(k_{1}, \ldots, k_{d l}\right)$, with $k_{i} \in \mathbb{N}_{0}^{+}$for $i=1 \ldots \ldots d$. For $\mathbf{n}, \mathrm{k} \in\left(\mathbb{N}_{0}^{+}\right)^{d}$ multi-integers we define,

$$
\mathbf{k} \leq \mathbf{n} \Leftrightarrow\left(k_{1} \leq n_{1} \wedge \cdots \wedge k_{d} \leq n_{d}\right) .
$$




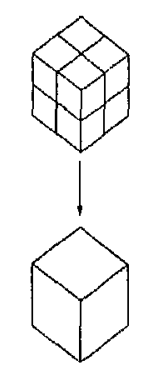

a. Full coarsening.

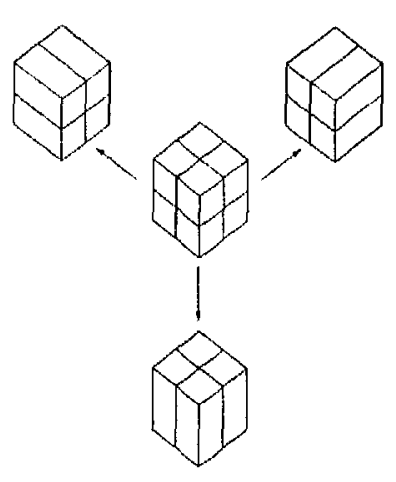

b. Multiple semi-coarsening.

Figure 1: Two types of $3 \mathrm{D}$ coarsenings.

In a similar way we define $\mathbf{k} \nless \mathbf{n}, \mathbf{k}=\mathbf{n}, \mathbf{k} \neq \mathbf{n}$, etc. Notice that $\mathbf{k} \neq \mathbf{n}$ means that all elements are unequal. The maximum of two multi-integers is defined as

$$
\max (\mathbf{k}, \mathbf{n})=\left(\max \left(k_{1}, n_{1}\right), \cdots, \max \left(k_{i}, n_{d}\right)\right)
$$

Further, we introduce the relation $\mathbf{k} \doteq \mathbf{n}$, and similarly $\mathbf{k}>\mathbf{n}$, by

$$
\mathbf{k} \doteq \mathbf{n} \Rightarrow\left(k_{1}=n_{1} \quad \vee \cdots \vee \quad k_{d}=n_{d d}\right) \text {. }
$$

We define a regular mesh $\Omega_{\mathbf{k}}$ on $\Omega$, with mesh sizes $h_{k}=2^{-k}=\left(2^{-k_{1}}, \ldots, 2^{-k_{l} l}\right)$ in the different coordinate directions. The volume of a grid cell $\Omega_{\mathrm{kj}} \in \Omega_{\mathbf{k}}, 0 \leq \mathbf{j} \leq 2^{\mathbf{k}}$ is denoted by $\left\|\mathbf{h}_{\mathbf{k}}\right\|=h_{k_{1}} \ldots h_{k_{d}}$ and $|\mathbf{k}|=k_{1}+\ldots+k_{i d}$ is called the level of grid $\Omega_{\mathbf{k}}$. The coarsest grid is $\Omega_{\mathrm{o}}$, with $\mathbf{o}=(0, \ldots, 0)$ and therefore the coarsest level is zero. The vertices of the grid $\Omega_{\mathbf{k}}$ are denoted by $\Omega_{\mathbf{k}}^{+}$, and $\Omega_{\mathbf{k}}^{+}$denotes the $\mathbf{i}$-th vertex on grid $\Omega_{\mathbf{k}}$, with $\mathbf{o} \leq \mathbf{i} \leq 2^{\mathrm{k}}$.

\subsection{Bases and spaces}

A function $u \in \mathcal{C}(\Omega)$ is approximated on $\Omega_{\mathrm{k}}$ by

$$
u \approx u_{\mathbf{k}}=\sum_{\mathbf{j}} u_{\mathbf{k j}} \varphi_{\mathrm{kj}}
$$

Here $\varphi_{\mathbf{k j}}$ is the usual piecewise $d$-linear basis function with $\operatorname{supp}\left(\varphi_{\mathbf{k j}}\right)=\left[\left(j_{1}-1\right) 2^{-k_{1}},\left(j_{1}+\right.\right.$ $\left.1) 2^{-k_{l}}\right] \times \cdots \times\left[\left(j_{d}-1\right) 2^{-k_{l l}},\left(j_{d l}+1\right) 2^{-k_{d}}\right]$ of tensor product type. The standard finite element basis $B_{\mathbf{k}}$ is defined as

$$
B_{\mathrm{k}}=\left\{\varphi_{\mathrm{kj}} \mid \mathbf{o} \leq \mathbf{j} \leq 2^{\mathrm{k}}\right\}
$$

and the corresponding space of piecewise $d$-linear functions is,

$$
V_{\mathrm{k}}=\operatorname{Span}\left(B_{\mathrm{k}}\right)
$$

The intersection of the support of two functions $\varphi_{\mathbf{k j}} \in B_{\mathbf{k}}$ and $\varphi_{\mathrm{ni}} \in B_{\mathbf{n}}$ is defined by

$$
\operatorname{Int}\left(\varphi_{\mathrm{kj}}, \varphi_{\mathrm{ni}}\right)=\operatorname{supp}\left(\varphi_{\mathrm{kj}}\right) \cap \operatorname{supp}\left(\varphi_{\mathrm{ni}}\right)
$$


The spaces $\left\{V_{k}\right\}$ form a partially ordered set [9], for which we can define the hierarchical space.

$$
W_{\mathbf{k}}=\operatorname{Span}\left(\left\{\tilde{\varphi}_{\mathbf{k j}}=\varphi_{\mathbf{k j}} \mid \begin{array}{lll}
j_{i} \text { odd, } \quad 0 \leq j_{i} \leq 2^{k_{i}}, & \text { if } k_{i} \geq 0 \\
j_{i}=0,1 & \text { if } \begin{array}{l}
k_{i}=0 \\
i=1, \ldots, d
\end{array}
\end{array}\right\}\right)
$$

Here $\tilde{\varphi}_{\mathrm{kj}}$ is a hierarchical basis function, i.e. the same function as the usual standard basis function, but only defined for $\mathbf{j}$, for which $\Omega_{\mathrm{kj}}^{+}$does not appear in any of the coarser gricls. In the following we will denote these hierarchical points with "j odd", although strictly spoken this is not true for the grids with $\|\mathbf{k}\|=0$. The hierarchical basis functions. specific for grid $\Omega_{\mathbf{k}}$, are defined by

$$
\tilde{B}_{\mathbf{k}}^{H}=\left\{\tilde{\varphi}_{\mathrm{kj}} \mid \tilde{\varphi}_{\mathrm{kj}} \in W_{\mathrm{k}}\right\} .
$$

The hierarchical basis for $V_{\mathbf{k}}$, which can be decomposed as $V_{\mathbf{k}}=\oplus_{\mathbf{o} \leq \mathbf{n} \leq \mathbf{k}} W_{\mathbf{n}}$, reads

$$
\tilde{B}_{\mathbf{k}}=\bigcup_{0 \leq \mathbf{n} \leq \mathbf{k}} \tilde{B}_{\mathbf{n}}^{H}
$$

Using this basis we are able to approximate a function $u$ on $\Omega$ by

$$
u \approx u_{\mathbf{k}}=\sum_{\mathrm{o} \leq \mathbf{n} \leq \mathrm{k}} \sum_{\mathbf{j} \in \Omega_{\mathbf{n}}} \tilde{u}_{\mathbf{n j}} \tilde{\varphi}_{\mathbf{n j}}
$$

where $\tilde{u}_{\mathbf{n} \mathbf{j}}$ are the hierarchical coefficients. Note that $\operatorname{Span}\left(\tilde{B}_{\mathbf{k}}\right)=\operatorname{Span}\left(B_{\mathbf{k}}\right)=V_{\mathbf{k}}$. It has been shown [9] that, for any $\mathbf{m}, \mathbf{o} \leq \mathbf{m} \leq \mathrm{e}=(1,1,1)$, the hierarchical coefficients for piecewise linear functions can be estimated by

$$
\left\|\tilde{u}_{\mathrm{kj}}\right\|_{2} \leq\left\|D^{(\mathbf{e}+\mathbf{m})} u\right\|_{2}\left\|\mathbf{h}_{\mathbf{k}}\right\|^{2} 2^{-i / 2} 3^{-|\mathbf{m}| / 2} \mathbf{h}_{\mathbf{k}}-(\mathbf{e}-\mathbf{m}) .
$$

We see that this bound depends essentially on the volume $\left\|\mathbf{h}_{\mathbf{k}}\right\|$ of the grid cells. Therrfore it seems reasonable to select approximating function spaces $W_{\mathbf{k}}$, and therefore the grids $\Omega_{\mathbf{k}}$ such that those with the smallest volume $\left\|\mathbf{h}_{\mathbf{k}}\right\|$ are omitted. In this way we obtain the sparse grid [18]. The hierarchical basis for this sparse grid on level $\ell$ is denotod by

$$
\tilde{B}_{\ell}=\bigcup_{0 \leq|\mathbf{n}| \leq \ell} \tilde{B}_{\mathbf{n}}^{H}
$$

and the corresponding function space by $V_{\ell}=\operatorname{Span}\left(\tilde{B}_{\ell}\right)$.

\subsection{The problem}

We ronsider the following linear elliptic problem

$$
\begin{array}{rlll}
-\nabla \cdot(\mathbf{a} \nabla u) & =f & \text { on } & \Omega, \\
\alpha(\mathbf{a} \nabla u) \cdot \mathbf{n}+\beta u & =\gamma & \text { on } & \Gamma .
\end{array}
$$

Here the data and the coefficients are functions on $\Omega$. For the FEM discretisation wo consider its variational form: find $u \in H^{\prime}(\Omega)$, such that $a(u, v)=f(v)$ for all $v \in H^{\prime}(\Omega)$. with

and

$$
a(u, v)=\int_{\Omega}(\nabla v)^{T} \mathrm{a} \nabla u d \Omega+\int_{\Gamma} \beta u v / \alpha d \Gamma
$$

$$
f(v)=\int_{\Omega} f v d \Omega+\int_{\Gamma} v \gamma / \sigma d \Gamma
$$

A standard FEM on grid $\Omega_{\mathrm{k}}$ is obtained by selecting trial and test functions in $\operatorname{Span}\left(B_{\mathrm{k}}\right)$. This yiolds the discrete equations $\sum_{\mathbf{j}} a\left(\varphi_{\mathbf{k} \mathbf{j}}, \varphi_{\mathbf{k} \mathbf{i}}\right) u_{\mathbf{k j}}=f\left(\varphi_{\mathbf{k} \mathbf{j}}\right)$, which system is also do:noted in matrix-form by $A_{\mathrm{kk}} u_{\mathrm{k}}=f_{\mathrm{k}}$. 


\subsection{Discretisation of the hierarchical system}

To discretise equation (1) on the sparse grid, we use $\tilde{B}_{p}$ as the basis for test and trial functions:

$$
u_{\ell}=\sum_{\mathbf{0} \leq|\mathbf{n}| \leq \ell} \sum_{\mathbf{j} o \mathrm{dd}} \tilde{u}_{\mathbf{n j}} \tilde{\varphi}_{\mathbf{n} \mathbf{j}}
$$

Substitution in (2) and (3) yields the equations

$$
\sum_{0 \leq|\mathbf{n}| \leq l} \sum_{\mathbf{j}} a\left(\tilde{\varphi}_{\mathbf{n j}}, \tilde{\varphi}_{\mathbf{k i}}\right) \tilde{u}_{\mathbf{n j}}=\left(f, \tilde{\varphi}_{\mathbf{k i}}\right)
$$

This system is called the hierarchical system and is denoted by

$$
\tilde{A}_{\rho} \tilde{u}_{p}=\tilde{f}_{\ell} \text {. }
$$

The matrix $\tilde{A}_{\ell}$ consists of blocks $\left(\tilde{A}_{\mathbf{n k}}\right)_{0 \leq \mid \mathbf{n}\},|\mathbf{k}| \leq \ell}$ where $\tilde{A}_{\mathbf{n k}}$ consists of coefficients $\left(a\left(\tilde{\varphi}_{\mathbf{n} \mathbf{j}}, \tilde{\varphi}_{\mathbf{k i}}\right)\right)$. The blocks $\tilde{A}_{\mathbf{k} \mathbf{k}}$ on the diagonal of $\tilde{A}_{\ell}$ are diagonal blocks, because $\operatorname{Int}\left(\tilde{\varphi}_{\mathbf{k j}}, \tilde{\varphi}_{\mathbf{k i}}\right)=\emptyset$ cxcept for $\mathbf{i}=\mathbf{j}$. In general, the functions $\tilde{\varphi}_{\mathbf{n j}}$ and $\bar{\varphi}_{\mathbf{k i}}$ can live on completely independent grids $\Omega_{\mathrm{n}}$ and $\Omega_{\mathrm{k}}$ and there is no obvious and efficient technique to calculate these matrix entries as is the case for standard finite elements. Especially, if we consider equations with variable coefficient a, the efficient computation of these integrals is not straightiforward. To avoid the problem of explicit calculation of $a\left(\tilde{\varphi}_{\mathrm{nj}}, \tilde{\varphi}_{\mathbf{k i}}\right)$ we want to derive the discrete equations from the usual FEM stiffness matrices $A_{\mathbf{k k}}$.

For $\mathbf{k} \leq \mathbf{m}$ let $R_{\mathrm{km}}: V_{\mathrm{m}} \rightarrow V_{\mathbf{k}}$ be the restriction defined by interpolation at noclal points $\Omega_{\mathbf{k}}^{+}$, then every function $\tilde{\varphi}_{\mathbf{k i}} \in \tilde{B}_{\mathbf{k}}^{I I}$ is represented on grid $\Omega_{\mathrm{m}}$ by

$$
\tilde{\varphi}_{\mathbf{k i}}=\sum_{\mathbf{p}} R_{\mathrm{km}, \mathrm{ip}} \varphi_{\mathrm{mp}}
$$

Now, the left-hand side in (5) reads, with $m=\max (k, \mathbf{n})$,

$$
\begin{aligned}
& \sum_{0 \leq|\mathbf{n}| \leq \ell} \sum_{\mathbf{j}} a\left(\tilde{\varphi}_{\mathbf{k i}}, \tilde{\varphi}_{\mathbf{n j}}\right) u_{\mathbf{n j}} \\
& =\sum_{0 \leq|\mathbf{n}| \leq \ell} \sum_{\mathbf{j}} a\left(\sum_{\mathrm{p}} R_{\mathrm{km}, \mathbf{i p}} \varphi_{\mathrm{mp}}, \sum_{\mathbf{q}} R_{\mathbf{n m}, \mathbf{j} \mathbf{q}} \varphi_{\mathbf{m q}}\right) u_{\mathbf{n j}} \\
& =\sum_{\mathbf{n}} \sum_{\mathbf{j}, \mathrm{p}, \mathrm{q}} R_{\mathrm{km}, \mathrm{ip}} R_{\mathrm{nm}, \mathbf{j} \mathbf{q}} A_{\mathrm{mm}, \mathrm{pq}}{ }^{\prime \prime \prime} \mathbf{\mathrm { j }} \\
& =\sum_{0 \leq|\mathrm{n}| \leq \ell}\left(R_{\mathrm{km}} A_{\mathrm{mm}} R_{\mathrm{nm}}^{7^{\prime}}\right) u_{\mathrm{n}} \\
& =A_{\mathbf{k k}} u_{\mathrm{k}}+\sum_{\mathbf{n}>\mathbf{k},|\mathbf{m}| \leq \ell}+\sum_{\mathbf{n}>\mathbf{k},|\mathbf{m}|>\ell}\left(R_{\mathbf{k m}} A_{\mathrm{mm}} R_{\mathrm{nm}}^{T}\right) u_{\mathbf{n}} .
\end{aligned}
$$

Thus we express the residual computation for the equations (5) in terms of the uswal FEM stiffness matrices $A_{\mathbf{k k}}$. The efficient calculation of $(6)$ is our immediate concern. Below we restrict ourselves to the constant coefficient case.

\subsection{Semi-orthogonality}

If for the Poisson equation $a\left(\tilde{\varphi}_{\mathbf{n j}}, \tilde{\varphi}_{\mathbf{k i}}\right)$ vanishes, the functions $\tilde{\varphi}_{\mathbf{n j}}$ and $\tilde{\varphi}_{\mathbf{k i}}$ are callod semi-orthogonal [15]. For the efficient computation of the hierarchical system this is a useful property, because each semi-orthogonality relation contributes with a zero entry in 
the hierarchical stiffness matrix. In [2] it is shown that this semi-orthogonality property holds for many functions $\tilde{\varphi}_{\mathrm{nj}} \in \tilde{B}_{\mathrm{n}}^{H}$ and $\tilde{\varphi}_{\mathrm{ki}} \in \tilde{B}_{\mathrm{k}}^{H}$. Below we will discuss this property for the two- and three-dimensional case separately, because there is a significant difference between both cases.

Two-dimensional case. For the Poisson equation, in the two-dimensional case [2] we have, $a\left(\tilde{\varphi}_{\mathbf{n j}}, \tilde{\varphi}_{\mathbf{k i}}\right)=0$ if $\mathbf{n} \neq \mathbf{k}$, because, considering a single derivative from $a\left(\tilde{\varphi}_{\mathbf{n j}}, \tilde{\varphi}_{\mathbf{k i}}\right)$,

$$
\int_{\Omega}\left(\frac{\partial \tilde{\varphi}_{\mathbf{n j}}}{\partial x_{p}} \frac{\partial \tilde{\varphi}_{\mathbf{k i}}}{\partial x_{p}}\right) d \Omega \neq 0 \Leftrightarrow\left(n_{p}=k_{p}\right) \wedge\left(j_{p} 2^{n_{p}}=i_{p} 2^{k_{p}}\right) .
$$

Hence functions on grids $\Omega_{\mathbf{n}}$ and $\Omega_{\mathbf{k}}$ with $\mathbf{n} \neq \mathbf{k}$ are semi-orthogonal.

For the functions $\tilde{\varphi}_{\mathbf{n j}}$ and $\tilde{\varphi}_{\mathbf{k i}}$ with $\mathbf{k} \doteq \mathbf{n}$ we introduce $\mathbf{m}=\max (\mathbf{k}, \mathbf{n})$, then, because $d=2$, either $\mathbf{m}=\mathbf{n}$ or $\mathbf{m}=\mathbf{k}$. Therefore, the bilinear form can be calculated by (6) and, because $|\mathbf{m}| \leq \ell$ is always ensured, this means that the third term in equation (6) can be dropped. Below, we will see that this is not true in the three-dimensional casc.

What remains is the efficient matrix vector multiplication. With precalculated values of $A_{\mathbf{k k}},|\mathbf{k}| \leq \ell$, we use (6) for a residual computation of (5). One can readily verify that for the constant coefficient case the total number of operations for the matrix vector multiplication (5) is proportional to $O\left(\ell^{2} 2^{\ell}\right)$ for $O\left(\ell 2^{\ell}\right)$ standard basis points. Therefore the method is suboptimal.

Three-dimensional case. Also in the 3D case we have semi-orthogonality for functions $\tilde{\varphi}_{\mathbf{n j}}$ and $\tilde{\varphi}_{\mathbf{k i}}$ with $\mathbf{n} \neq \mathbf{k}$. The nonzero contributions in the stiffness matrix originate only from functions $\tilde{\varphi}_{\mathbf{n j}}$ and $\tilde{\varphi}_{\mathbf{k i}}$ with $\mathbf{n} \doteq \mathbf{k}$. This corresponds with grids $\Omega_{\mathbf{n}}$ and $\Omega_{\mathbf{k}}$ in the same coordinate plane in the grid of grids (see Figure 2). For $\phi_{\mathbf{k i}}$ (k fixell) a non-semi-orthogonal function $\tilde{\varphi}_{\mathrm{nj}}$ can live on any grid $\Omega_{\mathbf{n}}$ in one of the indicated plancs.

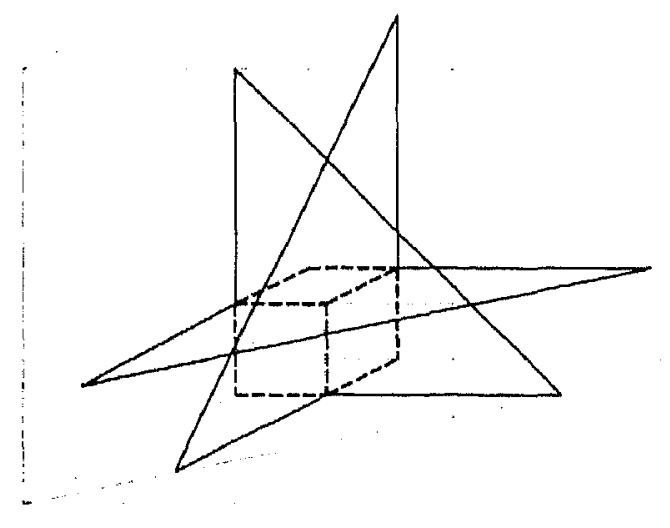

Figure 2: Planes with non-semi-orthogonal functions

Planes, in the space of grids, for which $\mathbf{k} \doteq \mathbf{n}$, for fixed $\mathbf{n}=(n, n, n)$. The diagomal plane corresponds with the grids $|\mathbf{k}|=n$.

In (6) we showed how we can calculate the contributions of $a\left(\tilde{\varphi}_{\mathrm{nj}}, \tilde{\varphi}_{\mathrm{ki}}\right)$ via the maximum grid. In the $3 \mathrm{D}$ case one can verify that, different from $2 \mathrm{D}$, with $|\mathbf{n}| \leq \ell,|\mathbf{k}| \leq \ell$, 
the relations $a\left(\tilde{\varphi}_{\mathbf{n} \mathbf{j}}, \tilde{\varphi}_{\mathbf{k i}}\right) \neq 0$ and $\mathbf{m}=\max (\mathbf{k}, \mathbf{n})$ do not imply $|\mathbf{m}| \leq \ell$. I.e. the maximum grid $\Omega_{\mathrm{m}}$ is not always part of the sparse grid of grids. In fact, we see that in the 3D case we have $|\mathrm{m}| \leq 2 \ell$. This implies that application of (6) requires the evaluation of FEM stiffness matrices on level $2 \ell$. We call the approach of using the grids up to level $2 \ell$ the semi-sparse technique. Here we need the evaluation of $\mathcal{O}\left(\ell^{2}\right)$ stiffness matrices of order $\mathcal{O}\left(2^{2 \ell}\right)$. This is well between the sparse grid technique with $\mathcal{O}\left(\ell^{2}\right)$ stiffness matrices of order $\mathcal{O}\left(2^{\ell}\right)$ and the full grid technique with one $\mathcal{O}\left(3^{\ell}\right)$ stiffness matrix. In Section 3 we will see that a similar combination of grids can be useful for the solution of more complex non-linear systems.

At first sight it seems an alternative not to compute the residual of equation (6) exactly, but to introduce an approximation of the matrix $\tilde{A}_{p}$ by taking the expression (6) in which the terms with $|\mathrm{m}|>\ell$ are omitted. One can verify that all discarded entries from the matrix $\tilde{A}_{\ell}$ are of relatively small size. In this way we obtain an approximated stiffness matrix, denoted as $\hat{A}_{\ell}$. One might expect that this $\hat{A}_{\ell}$ could still be some sufficiently accurate approximation of the discrete Laplacian. However, it can be shown that this approximate stiffness matrix $\hat{A}_{\ell}$ is not useful for further computations becanse it is not positive definite. In Table 1 we show the extreme eigenvalues of $\hat{A}_{p}$ and $\tilde{A}_{p}$ for various $\ell$. The smallest eigenvalue of $\hat{A}_{\ell}$ becomes negative, which ruins one of the essential properties of $A_{\ell}$.

\begin{tabular}{|r|c|r|r|r|}
\cline { 3 - 5 } \multicolumn{2}{c|}{} & $\ell=4$ & $\ell=5$ & $\ell=6$ \\
\hline$\tilde{A}_{\ell}$ & $\lambda_{\max }$ & 2.2843 & 3.3898 & 4.7756 \\
\cline { 2 - 5 }$\tilde{A}_{\ell}$ & $\lambda_{\min }$ & 0.6323 & 0.1803 & 0.0582 \\
\hline$\hat{A}_{\ell}$ & $\lambda_{\max }$ & 2.1498 & 3.1085 & 4.3162 \\
\cline { 2 - 5 }$\tilde{A}_{\ell}$ & $\lambda_{\min }$ & 0.5168 & -0.0251 & -0.1283 \\
\hline
\end{tabular}

Table 1: Extreme eigenvalues for $\tilde{A}_{\rho}$ and $\hat{A}_{\rho}$.

Following the semi-sparse-grid approach, one can verify that in the constant coefficiont case we can perform a residual computation with order $O\left(4^{\ell}\right)$ operations for $O\left(f^{2} 2^{f}\right)$ sparse grid points. Of course the additional work and also the extra storage is a disadvantage of this method. As a compromise between the sparse and the semi-sparse-gricl approach one might consider discretisations which are obtained by not using all grids uj)

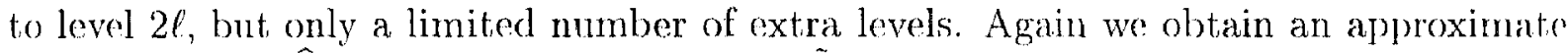
stiffness matrix $\hat{A}_{\ell}$, with a lower accuracy as $\tilde{A}_{\ell}$, but the oporation count will be smallor. However one should be very careful with this approach, since the possibility exists that $\hat{A}_{p}$ becomes indefinite if an insufficient number of arditional lovels is taken into account.

\section{The Euler equations for 3D CFD}

In this section we consider the multigrid solution of the steady, 3D Euler equations of gas dynamics. The equations are discretised in their integral form. The computational domain $\Omega$ is divided, in a regular manner, in coll-centered finite volumes. These finite volumes are arbitrarily shaped hexahedra. Following the Godunov approach, along each coll face the flux vector is assumed to be constant and determined by a uniformly constant left and right state. To solve the resulting 1D Riemann problem over the coll face for a non-isenthalpic perfect-gas flow, we apply the $3 \mathrm{D}$ extension of the $2 \mathrm{D}$ P-variant $[7]$ of Osher's approximate Riemann solver. For the left and right cell-face states, we tike 
the first-order accurate approximations. At a later stage, these approximations will be replaced by higher-order accurate ones, in which case also limiters are introduced. Wo emphasize that the major challenge is to know how to solve first-order accurate discrete, steady 3D Euler equations at efficient, grid-independent convergence rates. Once this is known, solving higher-order accurate discrete, steady 3D Euler equations can be done by a standard procedure, e.g. by a defect correction method as outer and the efficient multigrid method as inner iteration [10].

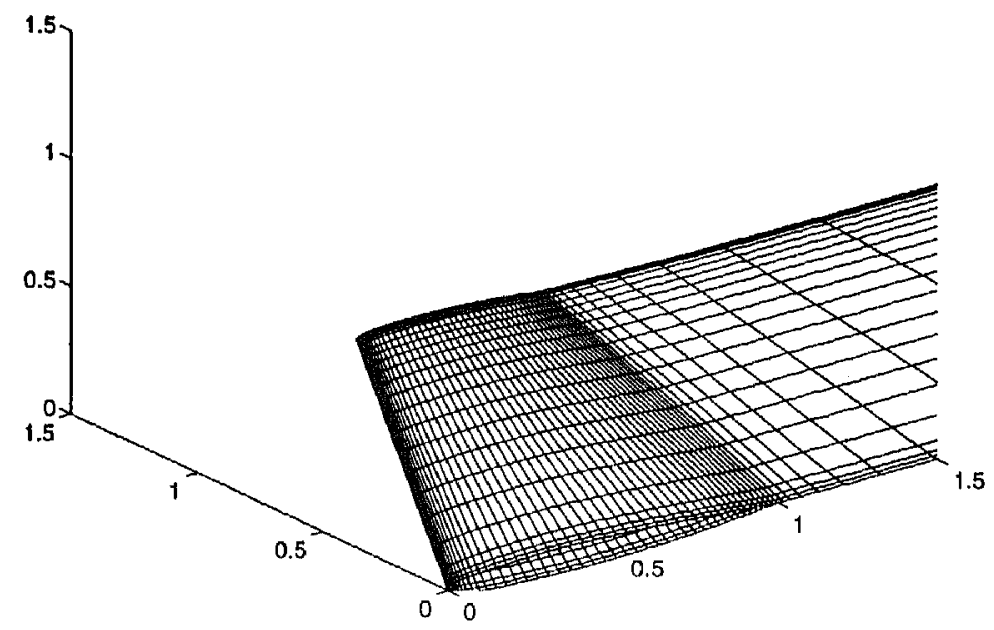

a. At upper side half-wing.

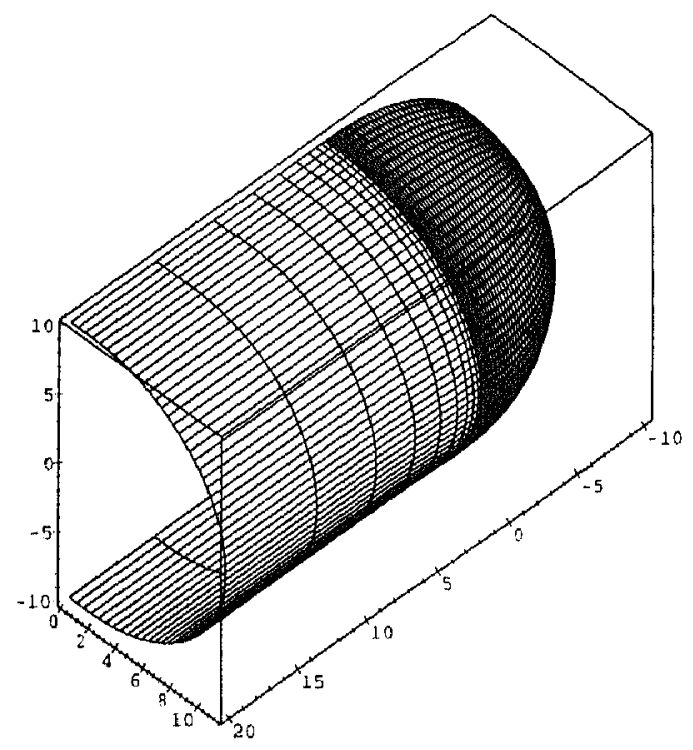

b. At far-field bounclary.

Geometry of the mesh around the ONERA-M16 half-wing. 


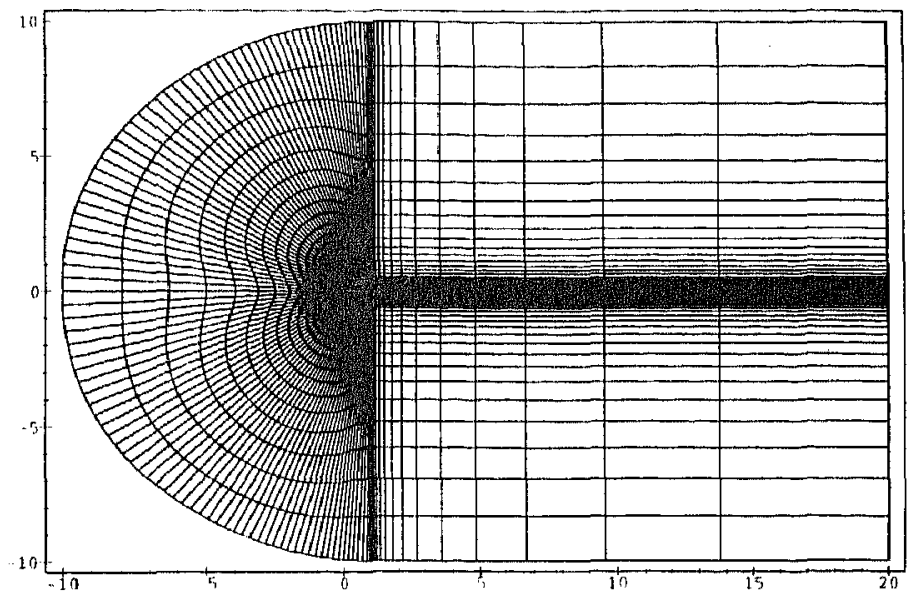

c. At symmetry boundary.

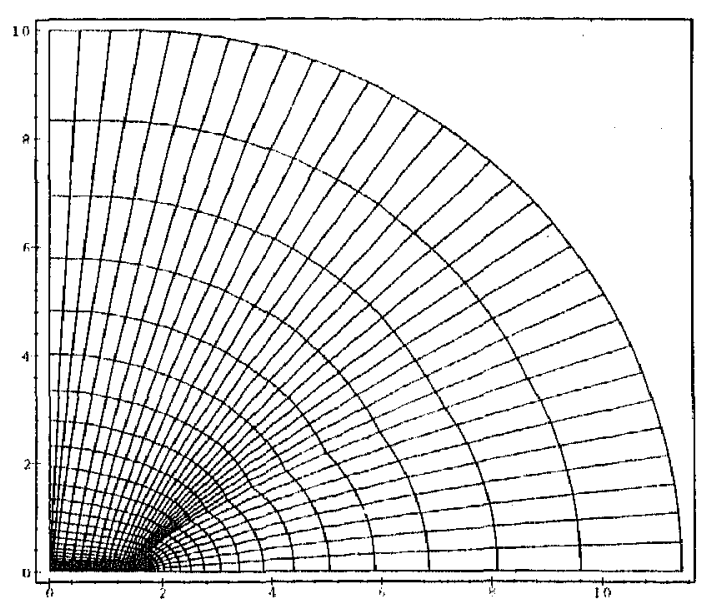

d. At uppor part downstream boundary.

Figure 3: Views at $128 \times 32 \times 32$ C-()-type gricl ONERA-M6 half-wing. 


\subsection{Standard multigrid}

\subsubsection{The method}

First we briefly describe the standard 3D multigrid algorithm on which our methor is built. We use the 3D generalization of the optimal 2D multigrid approach, that was described in $[7,6]$. As the smoothing technique for the first-order discrete Euler equations, we apply collective symmetric point Gauss-Seidel relaxation. The four different symmetric relaxation sweeps that are possible on a regular $3 \mathrm{D}$ grid, are performed alternatingly. At each volume visited during a relaxation sweep, the system of five nonlinear equations is solved by Newton iteration.

As the standard multigrid method we apply the nonlinear version (FAS, [1]), preceded by nested iteration (FMG, [1]). For this we construct a nested set of grids such that each finite volume on a coarse grid is the union of $2 \times 2 \times 2$ volumes on the next finer gricl. Let $\Omega_{0}, \Omega_{1}, \ldots, \Omega_{\ell_{\max }}$ be the sequence of such nested grids ${ }^{1}$, with $\Omega_{0}$ the coarsest and $\Omega_{\ell_{\operatorname{tmax}}}$ the finest grid. Then, nested iteration is applied to obtain a good initial solution on $\Omega_{\ell_{\max }}$, whereas nonlinear multigrid is applied to converge to the solution on the fincst. grid, $q_{\ell_{\max }}$. The first iterate for the nonlinear multigrid cycling is the solution obtained by nested iteration. We proceed discussing both stages in more detail.

Nested iteration. The nested iteration starts with a user-defined initial estimate for $q_{0}$, the solution on the coarsest grid. To obtain an initial solution on a finer grid $\Omega_{r_{+1}}$, first the solution on the coarser grid $\Omega_{\ell}$ is improved by a single nonlinear multigrid cycle. Hereafter, this solution is interpolated to the finer grid $\Omega_{\ell+1}$. These steps are repeated until the highest level (finest grid $\Omega_{\ell_{\max }}$ ) has been reached.

Nonlinear multigrid iteration. Let $N_{\ell}\left(q_{\ell}\right)=0$ denote the nonlinear system of firstorder accurate discretised equations on $\Omega_{\ell}$, then a single nonlinear multigrid cycle is recursively defined by the following steps:

1. Improve on $\Omega_{\ell}$ the latest obtained solution $q_{\ell}$ by application of $n_{\text {pre relaxation }}$ sweeps.

2. Compute on the next coarser grid $\Omega_{\ell-1}$ the right-hand side $r_{\ell-1}=N_{\ell-1}\left(q_{\ell-1}\right)-$ $R_{\ell-1, \ell} N_{\ell}\left(q_{\ell}\right)$, where $R_{\ell-1, \ell}$ is a restriction operator for right-hand sides.

3. Approximate the solution of $N_{\ell-1}\left(q_{\ell-1}\right)=r_{\ell-1}$ by the application of $n_{\text {FAs }}$ nonlinear multigrid cycles. Denote the approximation obtained by $\tilde{q}_{\ell-1}$.

4. Correct the current, solution by: $q_{\ell}=q_{\ell}+P_{\ell, \ell-1}\left(\bar{q}_{\ell-1}-q_{\ell-1}\right)$, where $P_{\ell, \ell-1}$ is a prolongation operator for solutions.

5. Improve $q_{f}$ by application of $n_{\text {pest }}$ relaxations.

Steps (2),(3) and (4) form the coarse-grid correction. The restriction $R_{\ell-1, \ell}$ and the prolongation $P_{\ell, \ell-1}$ are the usual operators that are consistent with the piecewise constant approximation (for more details see [11]).

Notice that $\Omega_{\ell}$ in the classical seruence is denoted as $\Omega_{\rho_{e}}$ in the context of partially ordered grids. The approximating function spaces for the discrete Euler equations are

\footnotetext{
${ }^{1}$ Cosing the more complex notation of Section 2 , the sequence is denoted as $\Omega_{\mathrm{o}}, \Omega_{\mathbf{e}}, \ldots, \Omega_{\mathrm{f}_{\max } \mathrm{e}}$, with
$=(1,1,1)$. $e=(1,1,1)$
} 
piecewise constant functions on $\Omega_{p_{e}}$. Approximating properties for these functions have been studied in [9]. Since the MG method applies the prolongations and restrictions that are consistent with the approximation used, the corresponding function spaces $V_{\mathbf{k}}$ form a partially ordered set of tensor product type as treated in [9].

\subsubsection{Numerical results}

In this section we present convergence results obtained when solving discrete, steady perfect-gas Euler equations for a standard 3D transonic test case, the ONERA M6 halfwing at $M_{\infty}=0.84, \alpha=3.06^{\circ}$. A C-O-type grid is used, for which we give some views on the $128 \times 32 \times 32$ version in the Figures $3 a-3 d$.

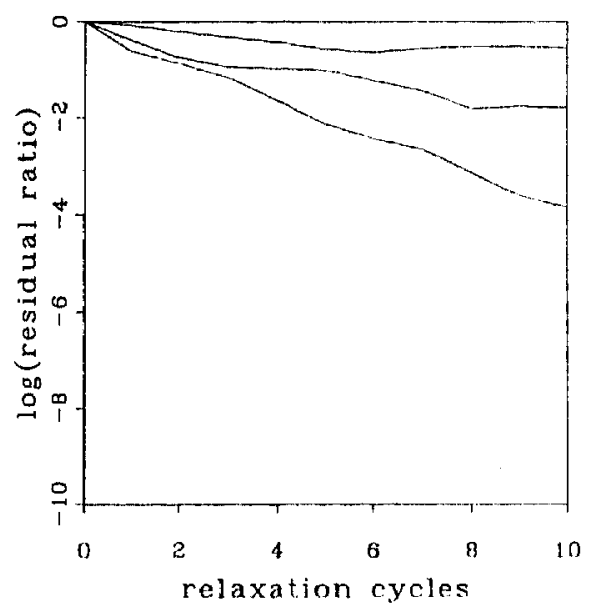

a. Single-grid.

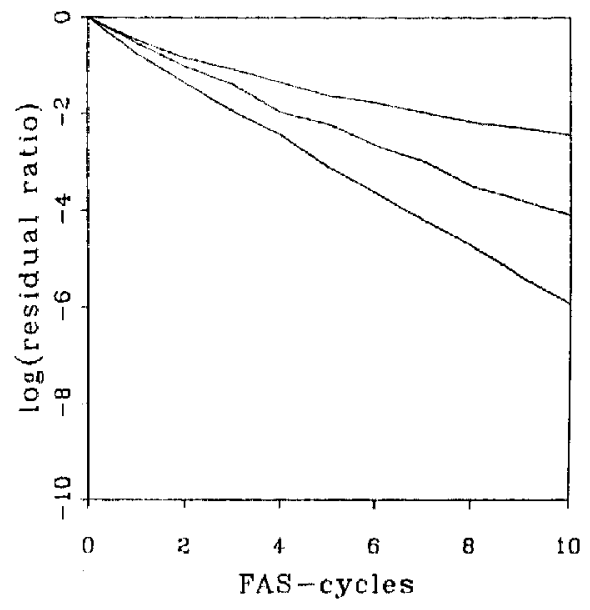

b. Standard multigrid.

Figure 4: Convergence behaviors of two solution methods, ONERA-M6 half-wing at $M_{\infty}=0.84, \alpha=3.06^{\circ}, \Omega_{\ell_{\max }}=(8 \times 2 \times 2) \times 2^{\ell_{\max }}-\operatorname{gricl}, \ell_{\max }=1,2,3$.

The slower convergence is for finer meshes.

In Figure 4 convergence results are given for single grid relaxation and for the stanclard multigrid method described above. In both graphs, the residual ratio is defincd as $\left\|R^{i}\right\|_{L_{1}} /\left\|R^{1}\right\|_{L_{1}}$, where $R^{i}$ is the mass defect of the discrete Euler equations and where $i$ refers to the status after the $i$-th iteration. For the standard multigrid convergence results shown in Figure $4 \mathrm{~b}$, we took $n_{\text {pre }}=0, n_{\text {post }}=1$, i.e. we applied sawtoothcycles. Though -of course- to a lesser extent than the single-grid convergence rosults (Figure 4a), the standard multigrid method's convergence results (Figure 4b) appear to be rather grid-dependent. We see that the convergence behaviour of the standard 3D algorithm is disappointing when compared to the same multigrid method's convergence rates for a 2D transonic test case [7]. An improvement to this might be found in deriving a more powerful smoother, keeping the other components of the numerical methorl the same. For reasons explained above, a more natural cure is not to apply standard full coarsening, but to use a multiple semi-coarsening or a sparse-grid algorithm insteat. 


\subsection{Multiple semi-coarsened multigrid}

\subsubsection{The method}

In this method, to solve the discrete problem on $\Omega_{\ell_{\text {max }} \mathbf{e}}$, we use the full family of grids $\left\{\Omega_{\mathbf{k}}\right\}$ with $\mathbf{o} \leq \mathbf{k} \leq \ell_{\max } \mathbf{e}$. For each $\Omega_{\mathbf{k}}, \mathbf{k}>\mathbf{o}$, three semi-coarsened grids are available. Figures $1 \mathrm{a}$ and $1 \mathrm{~b}$ show $3 \mathrm{D}$ standard coarsening and multiple semi-coarsening, respectively. Though multigrid with multiple semi-coarsening is expected to be most fruitful for $3 \mathrm{D}$ problems, as far as we know, applications have only been published for 2D. Pioneering work has been done by Mulder [13], who has introduced multiple semi-coarsening to overcome the poor convergence results observed in computing nearly grid-aligned flows governed by the steady, 2D Euler equations. In [16], Radespiel and Swanson continuc. research on Mulder's approach for the steady, 2D Euler equations. In the present paper we apply semi-coarsened multigrid to the steady, 3D Euler equations, and we pay particular attention to the different prolongation operators that can be used.

Also in the case of the semi-coarsened multigrid method we use FAS as the basic: multigrid algorithm, and on each grid we apply collective symmetric point Gauss-Scirlel relaxation as the smoothing technique. In the semi-coarsened multigrid method, howerer, wo replace the sequentially ordered set of grick $\left\{\Omega_{\ell \mathbf{e}}\right\}, \ell=0, \ldots, \ell_{\max }$, by a partially ordered set of grids $\left\{\Omega_{\mathbf{n}}\right\}, \mathrm{o} \leq \mathrm{n} \leq \mathrm{l}_{\max }$, with $\Omega_{\mathrm{o}}$ the coarsest and $\Omega_{\mathbf{I}_{\max }}$ the finest grirl. Now $|\mathbf{n}|$ is the level of grid $\Omega_{\mathbf{n}}$. The nesting and the semi-coarsening relation between these grids is described in [9]. The implementation is described in detail in $[8,11]$.

Nested iteration. Also in the semi-coarsening algorithm nested iteration (FMC $i$ ) is applied to obtain a good initial solution on the finest grid. We proceed discussing the present nested iteration and nonlinear multigrid iteration procedures in more detail. Tho nested iteration starts with a user-defined initial estimate on the coarsest grid, $\Omega_{\mathbf{o}}$, which is improved by relaxation. Next, the following two options can be used to continue the nested iteration:

- Grid-uise nested iteration. The approximate solution $q_{\mathrm{o}}$ is interpolated to all grids $\Omega_{\mathbf{k}}$ with $\mathrm{o} \leq \mathrm{k} \leq \mathrm{e}$, with the $3 \mathrm{D}$ prolongation according to formula (29) in [5]. (Sec. [11] for the implementation in the present. 3D Euler context.) Next, the solution $q_{\mathrm{e}}$ is improved by a single nonlinear multigrid cycle and prolongated to all grids $\Omega_{\mathbf{k}}$ with $\mathbf{k} \leq 2 \mathrm{e}$. Then, the above process can be repeated up to and inchuding level $d \ell_{\max }$. Notice that approximate solutions are only computed at the grids $\Omega_{\mathbf{o}}, \Omega_{\mathbf{e}}, \Omega_{2 \mathbf{e}}, \ldots$

- Level-by-level nested iteration. The approximate solution $q_{0}$ is interpolated to the three grids $\Omega_{1,0,0}, \Omega_{0,1,0}$ and $\Omega_{0,0,1}$ on the next level, with the same $3 \mathrm{D}$ prolongation mentioned above. Next, the three approximate solutions $q_{\mathbf{k}},|\mathbf{k}|=1$ are first improved by a single nonlinear multigrid cycle and then interpolated to all six grids $\Omega_{\mathbf{k}},|\mathbf{k}|=2$, on the next level. This process is repeated up to and including level $d \ell_{\max }$. Here, in contrast with to the previous strategy, solution improvements are made on all grids, level-by-level.

Nonlinear multigrid iteration. A single noulinear multigrid cycle on level $\ell$ is recursively defined by the following steps: 
1. For all grids $\Omega_{\mathbf{k}}$ at the next coarser level $|\mathbf{k}|=\ell-1$, that satisfy $\mathbf{k} \leq \ell_{\max } \mathrm{e}$, compute the same right-hand sides as in standard multigrid, but use as restriction operator the one described in [11].

2. Improve the approximate solutions on the coarser level $\ell-1$ by the application of a single nonlinear multigrid cycle.

3. Correct, the current solutions on level $\ell$ by one of two possible correction prolongations. The first prolongation (defect dependent weights) is an extension to 3D and to systems of equations, of the prolongation introduced by Naik and Van Rosendale [14]. It uses prolongation weights that are proportional to the absolute values of the restricted defect components. The second correction prolongation (fixed weights) is the one proposed in [5, eq.(36)], it has a-priori known prolongation weights +1 or -1 .

4. Improve the solutions on level $\ell$ by the application of $n_{\text {post }}$ relaxation sweeps.

\subsubsection{Numerical results for different prolongations.}

As a test problem we consider again the ONERA-M6 half-wing at the transonic conditions $M_{\infty}=0.84, \alpha=3.06^{\circ}$. We first compare the two prolongations mentioned above: the one with defect-dependent weights and the one with fixed weights. Convergence results obtained are given in Figure 5. In the two graphs, the residual ratio is defined

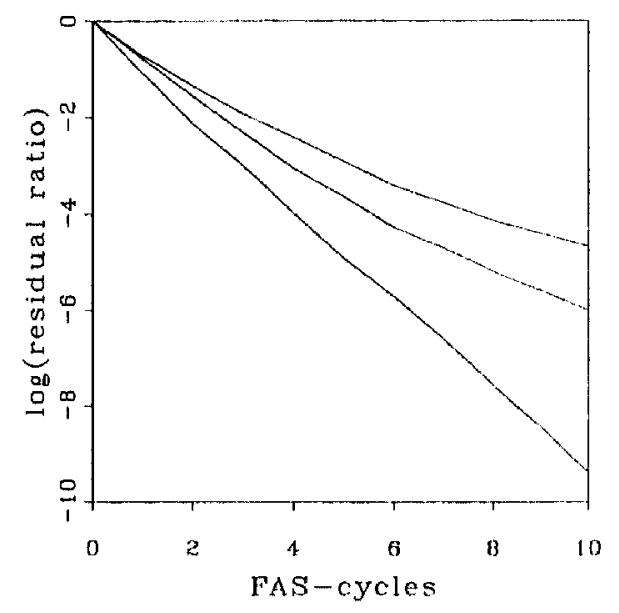

a. With defect-dependent prolongation weights.

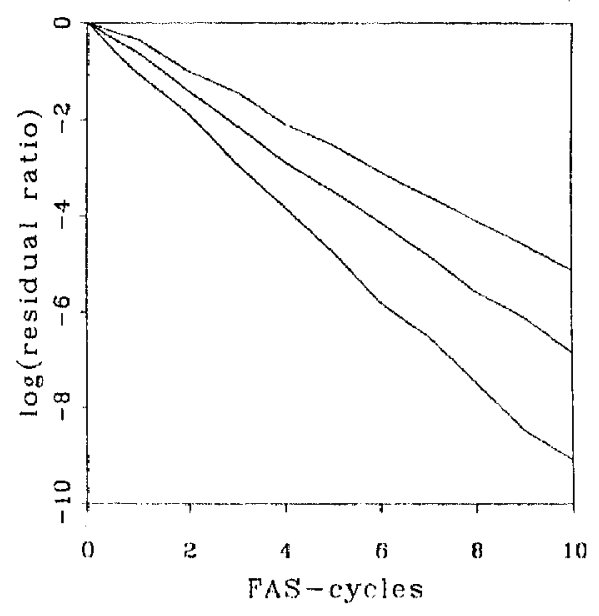

b. With fixed prolongation weights.

Figure 5: Convergence behaviors of two semi-coarsened miltigrid methods, ONERA-M6 half-wing at $M_{\infty}=0.84, \alpha=3.06^{\circ}, \Omega_{l_{\max }, m_{\max }, n_{\max }}=\left(8 \times 2^{l_{\max }}\right) \times\left(2 \times 2^{m_{\max }}\right) \times\left(2 \times 2^{n_{\max }}\right)$. grid, $l_{\max }=m_{\max }=n_{\max }=1,2,3$.

as $\left\|R^{i_{\text {FAS }}}\right\|_{L_{1}} /\left\|R^{1}\right\|_{L_{1}}$, where $R^{i_{\text {FAS }}}$ is the first component (i.e. the mass component) of the residual $N_{\ell_{\max } \mathrm{e}}\left(q_{\ell_{\max }}^{i_{\mathrm{FAS}}}\right)$, and where $i_{\mathrm{FAs}}$ refers to the status after the $i$-th FAS-cvele. Similar as for the standard multigrid convergence results (Figure $4 \mathrm{~b}$ ), here we also nsed sawtooth cycles $\left(n_{\text {pre }}=0, n_{\text {piost }}=1\right)$. The improvement of both semi-coarsened multigrid methods with respect to the standard imultigrid method is significant. Of both 
methods, the one with the fixed prolongation weights (Figure 5b) performs more than the one with defect-dependent prolongation weights (Figure 5a).

The convergence results may still be further improved. In Figure 6 we present results for the same solution strategy as that of Figure 5b, but now with V-cycles $\left(n_{\text {pre }}=n_{\text {post }}=\right.$ 1) and with the level-by-level nested iteration described in Section 3.2.1.

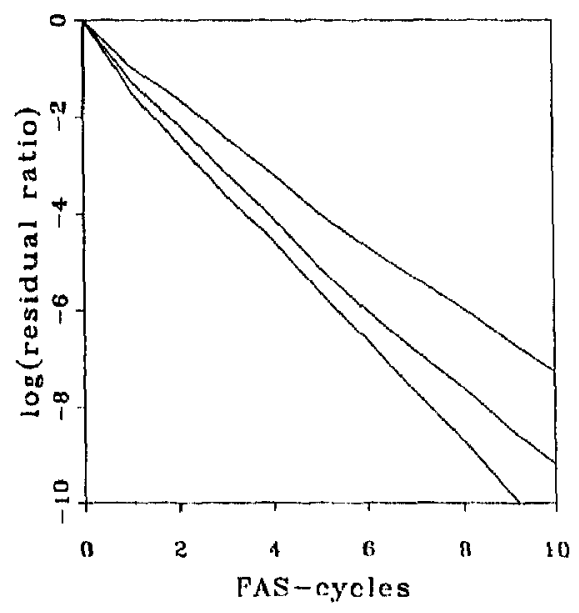

Figure 6: Convergence behaviour of semi-coarsened multigrid method with fixed prolongation weights, V-cycles and level-by-level nesterl iteration, for ONERA-M6 half-wing at $M_{\infty}=0.84, \alpha=3.06^{\circ}, \Omega_{l_{\max }, m_{\max }, n_{\max }}=\left(8 \times 2^{l_{\max }}\right) \times\left(2 \times 2^{m_{\max }}\right) \times\left(2 \times 2^{n_{\max }}\right)$ gridl, $l_{\max }=m_{\max }=n_{\max }=1,2,3$.

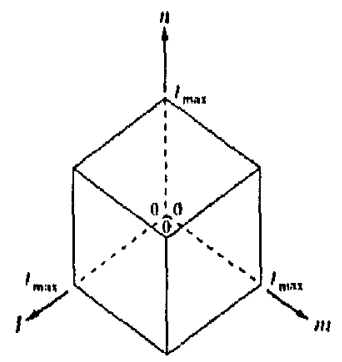

a. Full.

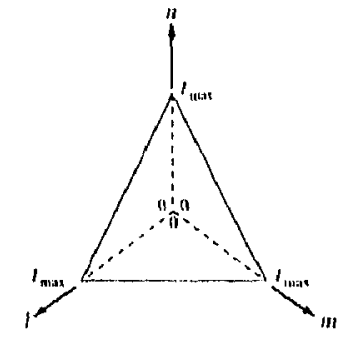

b. Sparse.

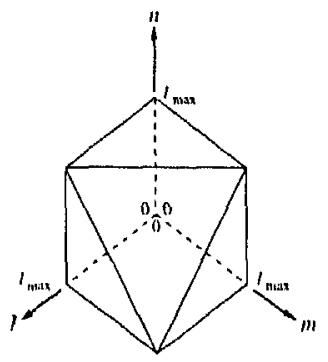

c. Semi-sparse.

Figure 7: Grids of grids.

\subsection{Sparse- and semi-sparse-grid multigrid}

\subsubsection{The methods}

The above multiple semi-coarsening methods for the Euler equations are methods for the solution for one system of discrete equations, defined in the 'finest' grid $\Omega_{1_{\operatorname{mnx}}}$ (we call it a full grid-of-grids semi-coarsening method), where all grids $\Omega_{\mathbf{k}}, \mathbf{0} \leq \mathbf{k} \leq \mathbf{1}_{\max }$ contribute to the solution process. A disadvantage of a full grid-of-grids semi-coarsening is that many grid cells are needed in total. With $N^{3}$ the total number of cells on the finest grid, in 3D, asymptotically standard multigrid nses $\frac{9}{8} N^{3}$ grid cells versus $8 N^{3}$ points for the full-grid-of-grids approach. An efficiency improvement can be achieved by thimning 
out the grid-of-grids. Then, if no 'finest grid' is available, accurate approximations can be constructed either by extrapolation (e.g. by Zenger's combination technique) or by the use of hierarchical bases. Most ambitious in this respect is the sparse gricl-ofgrids approach, where only grids $\Omega_{\mathbf{k}},|\mathbf{k}| \leq \ell_{\max }$, contribute (see [5, 4] and the further references there in). With the full grid-of-grids represented as a cube in Figure 7 a, the sparse grid-of-grids is the subset given in Figure $7 \mathrm{~b}$.

The reduction in the numbers of grid-cells is enormous. The computational complexity of the sparse grid-of-grids approach is $\mathcal{O}\left(N \log ^{2} N\right)$. Theoretically, the sparse grid-of-grids approach has the best ratio of discrete accuracy over number of grid points used [4]. In the ideal case the full grid-of-grids should be completely replaced by a sparse grid-of-grids. In practice, although very fast, the accuracy of the sparse grid approximations is slightly disappointing, and it appears that better accurate approximations are obtained not by only increasing the number of levels, but also by dropping the cells with extreme aspect ratios.

A compromise is the use of a semi-sparse grid-of-grids. This uses the family of grirls $\Omega_{\mathbf{k}},|\mathbf{k}| \leq 2 \ell_{\max }, \max _{i}\left|k_{r i}\right| \leq \ell_{\max }$, (see Figure $7 \mathrm{c}$ ), which (asymptotically) still has a computational complexity which is much smaller than that of the full-grid-of-grids approach, viz. $\mathcal{O}\left(N^{2} \log ^{2} N\right)$. Hence, though to a lesser extent than the genuine sparsegrid approach, it still is a cure to the 'curse of 3D'.

\subsubsection{Numerical results for sparse and semi-sparse multigrid.}

The numerical ingredients of both approaches are identical to those in the multiple semicoarsened multigrid method applied for obtaining Figure 6. Exactly the same level-bylevel method is applied, with as the only difference that in the sparse-grid casc tho multi-level semi-coarsening solver stops its work at level $\ell_{\max }$. From there the solution is extrapolated, by the combination techniquo as described e.g. in [17], to the vary finest grid, $\Omega_{\ell_{\text {max }} \text { e }}$ at level $3 \ell_{\text {max }}$. In the semi-sparse-grid approach the semi-coarsened multi-level algorithm is stopped at level $2 \ell_{\max }$ and from there, by the same combination technique, the finest-grid solution at $3 \ell_{\max }$ is computed. A particular advantage of the semi-sparse-grid approach as compared to the sparse-grid approach, is that the 3D extrapolation rule as proposed in [17] can be applied for all remaining grids, inclurling the grids along the boundaries of the grid-of-grids. In the sparse-grid approach this is not possible. There, for all boundary grids, i.e. $\Omega_{\mathbf{n}}$ for which $\|\mathbf{n}\| \equiv n_{1} \cdot n_{2} \cdot n_{3}=0$, onc has to introduce an additional extrapolation rule, e.g. by applying a $1 \mathrm{D}$ or a $2 \mathrm{D}$ combination extrapolation, which will inevitably result in some aclditional loss of accuracy. In the Figures $8 \mathrm{a}-\mathrm{c}$ we give an impression of the accuracy of the numerical solutions obtaincel by the different approaches for the ONERA wing problem. A reference solution is the fully converged $\mathcal{O}(h)$ finest grid solution Figure $8 \mathrm{c}$. This solution is the target for both solutions presented in Figures 8a-b. Of course, the semi-sparse grid solution (Figure

\begin{tabular}{llr}
\hline sparse & $\mathcal{O}\left(N \log ^{2} N\right)$ & 1 CPU time unit \\
semi-sparse & $\mathcal{O}\left(N^{2} \log ^{2} N\right)$ & 35 OPU time units \\
full & $\mathcal{O}\left(N^{3}\right)$ & 150 CPU time units \\
\hline
\end{tabular}

Table 2: Computing times for the solutions of the ONERA half-wing.

8b) comes closer to the reference solution. The sparse-grid solution (Figure 8a) is far 
a) sparse grid-of-grids
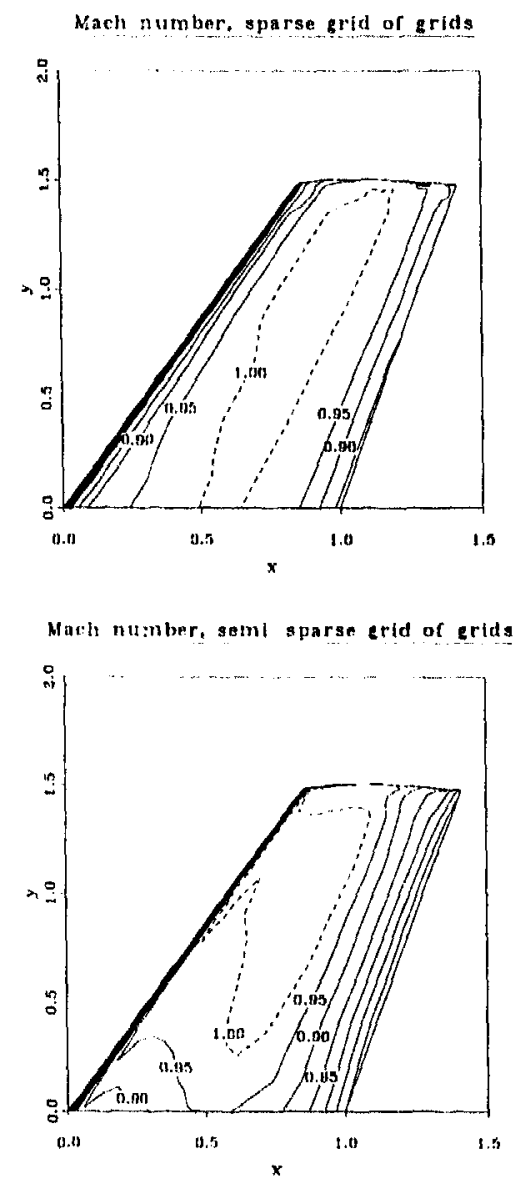

b) semi-sparse grid-of-grids

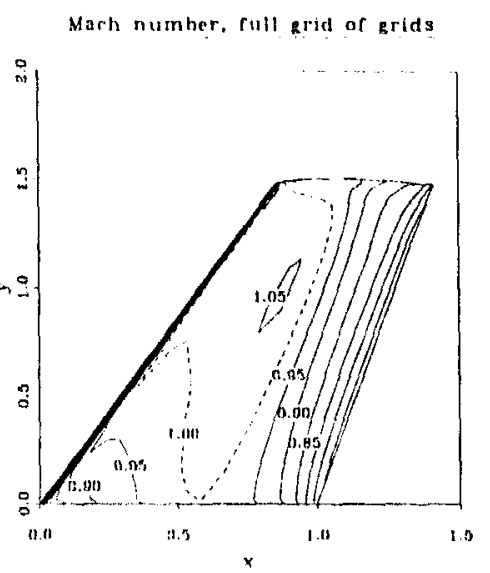

Figure 8: Mach number distribution on upper half-wing surface for different types of grid combinations. 
off, but it has been obtained at extremely low computational cost as compared to both the semi-sparse-grid approach and the full grid-of-grids approach. In Table 2 we give an impression of the relative computing times used.

\section{References}

[1] A. Brandt. Guide to multigrid development: In W. Hackbusch and U. Trottenberg, editors, Multigrid Methods, volume 960 of Lecture Notes in Mathematics, pages 220-312. Springer-Verlag, Berlin, 1982.

[2] H. J. Bungartz. Dünne Gitter und deren Anuendung bei der adaptiven Lösung der dreidimensionalen Poisson-Gleichung. PhD thesis, Institut für Informatik, TU München, 1992.

[3] M. Griebel, M. Schneider, and C. Zenger. A combination technique for the solution of sparse grid problems. In Proceedings of the IMACS International Symposium on. Iterative Methods in Linear Algebra, pages 263-281, Amsterdam, 1992. Elsevier.

[4] M. Griebel, C. Zenger, and S. Zimmer. Multilevel Gauss-Seidel-algorithms for full and sparse grid problems. Computing, 50:127-148, 1993.

[5] P. W. Hemker. Sparse-grid finite-volume multigrid for 3D-problems. Adv. Comput. Math., 4:83-110, 1995.

[6] P. W. Hemker and B. Koren. A non-linear multigrid method for the steady Euler equations. In A. Dervieux, B. van Leer, J. Périaux, and A. Rizzi, editors, Numericral Simulation of Compressible Euler Flows, volume 26 of Notes on Numerical Fluidl Mechanics, pages 175-196. Vieweg \& Son, Braunschweig, Germany, 1989.

[7] P. W. Hemker and S. P. Spckreijse. Multiple grid and Osher's scheme for the effirient solution of the steady Euler equations. Appl. Numer. Math., 2:458 476, 1986.

[8] P.W. Hemker and P.M. de Zeeuw. BASIS3, a data structure for 3-dimensional sparse grids. In H. Deconinck and B. Koren, editors, Euler and Navier-Stokes Solvers Usin! Multi-Dimensional Upunind Schemes and Multigrid Acreleration, volume 56 of Note's on Numerical Fluid Mechanics, pages 443-484. Vieweg, Braunschweig, 1996. In press.

[9] P.W. Hemker and C. Pflaum. Approximation on partially ordered sets of regular grids. Submited for publication, 1996.

[10] B. Koren. Defect correction and multigrid for an efficient and accurate computation of airfoil flows. J. Comput. Phys., 77:183-206, 1988.

[11] B. Koren, P.W. Hemker, and P.M. de Zeenw. Somi-coarsening in three directions for Euler-flow computations in three dimensions. In H. Deconinck and B. Koren, editors, Euler and Navier-Stokes Solvers Using Multi-Dimensional Upuind Schemes and Multigrid Acceleration, volume 56 of Notes on Numerical Fluid Mechanics, panges 547-567. Vieweg, Braunschweig, 1996. In press.

[12] C.B. Liem, T. Ln, and T.M. Shih. The Splitting Extrapolation Method. World Scientific, Singapore, 1995. 
[13] W. A. Mulder. A new multigrid approach to convection problems. J. Comput. Phys., 83:303-323, 1989.

[14] N. H. Naik and J. R. van Rosendale. The improved robustness of multigrid elliptic: solvers based on multiple semicoarsened grids. SIAM J. Numer. Anal., 30:215-229, 1993.

[15] C. Pflaum. A multi-level-algorithm for the finite-element-solution of general second order elliptic differential equations on adaptive sparse grids. Technical Report TUMI-9419, SFB-Bericht Nr. 342/12/94 A, Technische Universität München. Institut für Informatik, May 1994.

[16] R. Radespiel and R. C. Swanson. Progress with multigrid schemes for hypersonic flow problems. J. Comput. Phys., 116, 1995.

[17] U. Riide. Multilevel, extrapolation, and sparse grid methods. In Multigrid Methodls IV, Proceedings of the Fourth European Multigrid Conference, Amsterdam, July 69, 1993, P.W. Hemker and P. Wesseling eds. Volume 116 of ISNM, pages 281-294, Basel, 1994. Birkhäuser.

[18] C. Zenger. Sparse grids. In Parallel algorithms for partial differential equation.s: Proceedings of the Sixth GAMM-Seminar, Kiel, Jan. 1990, Notes on Numcrical Fluid Mechanies, vol. 31. Vieweg, Brannschweig, 1991. 